\title{
Corrupción y desarrollo: Deconstruyendo el discurso del Banco Mundial
}

\author{
Salvador Orlando Alfaro \\ Departamento de Sociología y Estudios Sociales \\ Universidad de Regina (Canadá)
}

\section{Introducción}

La corrupción es la nueva luminaria en el escenario del desarrollo. En el pasado fue considerada en los círculos oficiales del desarrollo como un tema para los especialistas académicos. En la actualidad, la "corrupción" de acuerdo a James Wolfensohn, Presidente del Banco Mundial, se ha constituido en "el mayor obstáculo al desarrollo económico equitativo". En el mismo sentido, después de haber sido identificada en el pasado como un "tabú", ahora es un tema al cual le fue asignado un presupuesto de 7.5 millones de dólares en el año financiero de 1999 por el Instituto del Banco Mundial (Tesh 1999:1).

¿Por qué el imaginario "corrupción” entra abruptamente en el foco de atención después de 1996? ¿Por qué precisamente en ese momento? ¿Qué es lo que se encuentra detrás de los programas de "corrupción" y "anti-corrupción"? ¿Y cómo este nuevo centro de atención pasa a formar parte de la amplia agenda del desarrollo?

Las prácticas de corrupción -en el contexto del desarrollo- han sido reconocidas por una variedad de organizaciones y actores. Entre 
éstos se incluyen organizaciones intergubernamentales, el sector no gubernamental, algunas compañías privadas que han introducido códigos de conducta y varios gobiernos alrededor del mundo. En la realidad, cada actor ha adoptado un enfoque diferente sobre este tipo de prácticas.

Considerando la importancia que reviste esta problemática y debido a su alto grado de trascendencia tanto teórica como práctica, el presente análisis se centrará exclusivamente en uno de los actores principales en el drama del desarrollo: el Banco Mundial (BM), y principalmente en la actitud que esta institución ha adoptado en los últimos años con respecto al "disfuncional” problema de la corrupción. El BM se constituye en un estudio de caso significativo porque es el actor más prominente en la "industria del desarrollo" (Ferguson 1990). Además, talvez más importante que su influencia sobre el destino económico de los países del Sur, el BM es el representante indiscutido del discurso dominante del desarrollo, por lo que condiciona fuertemente los "marcos de referencia" en el que los otros actores del desarrollo se reflejan discursivamente (Escobar 1995). Es preciso, entonces, considerar el papel dirigente del BM en la promoción de la agenda anticorrupción, tanto en sus propios programas como en su apoyo a los "esfuerzos internacionales" en los que se ven involucrados la mayor parte de gobiernos y sociedades civiles del mundo periférico.

Nuestro propósito es intentar descifrar la visión que tiene el BM sobre la corrupción en un contexto histórico específico e institucional y que por lo mismo nos permita descubrir sus presupuestos inherentes. En el desarrollo de la discusión se mostrará cómo el punto de vista del BM es parcial y que, por lo mismo, de ningún modo es universalmente aplicable y tampoco tiene un carácter neutral.

Idealmente, la desconstrucción del discurso debería ser acompañada por dos estudios posteriores. Estos podrían entrelazar el presente análisis discursivo con el mundo de la experiencia: primero, los obstáculos institucionales al interior del BM que dan forma a las reglas específicas del discurso; y segundo, los efectos que este discurso particular tiene sobre los "clientes" del BM, incluyendo las estrategias de resistencia e incorporación generada por estos actores. En todo caso, la experiencia de las gentes es la que en definitiva le da ímpetus al análisis discursivo. Reconociendo que estos dos enfoques están bastante 
alejados del alcance del presente esfuerzo y por lo tanto no desarrollados explícitamente, ejemplos de factores institucionales y las implicaciones que tiene sobre algunos gobiernos, sin embargo, se mencionarán a través de todo el texto.

\section{Enfoque teórico}

En el presente análisis se discute el fenómeno de la corrupción dentro de una variedad de tradiciones fuertemente establecidas y que se han empeñado en discutir críticamente las categorías del desarrollo y percibirlas como "discursos" — como "sistemas de afirmaciones que construyen un objeto" - en lugar de considerarlas como descripciones no problemáticas de una prentendida realidad (Parker 1992: 5). En términos generales, las bases epistemológicas del análisis de discurso se pueden encontrar en el pensamiento post-estructuralista (Derrida 1978). Dentro de esta perspectiva "lenguaje y discurso no son vistos como una reflexión de la realidad social, sino constituidos en ésta" (Escobar 1997: 51). El hecho de utilizar el enfoque discursivo de ninguna manera puede llevarnos a pensar que las actividades vinculadas con las "mordidas", la extorsión, la malversación y el nepotismo no existan en la realidad o que no tengan efectos reales y negativos en la vida de miles de millones de personas; los estudios políticos y económicos son cruciales para la comprensión del fenómeno en su totalidad (Rose-Ackerman 1999). En nuestro caso, sin embargo, la preocupación se centra principalmente en analizar los presupuestos discursivos sobre los que descansa el punto de vista dominante sobre las prácticas de corrupción y sobre el cual se basan muchos estudios e informes, especialmente aquellos llevados a cabo por el BM e instituciones afines. Nuestro interés reside en localizar los contextos históricos, socio-culturales e ideológicos de este punto de vista, y las influencias institucionales que contribuyen a configurar el espacio en donde se identifican, entre otros aspectos claves, cuáles preguntas son formuladas y qué respuestas son encontradas aceptables. En la actualidad, existen en el mundo académico diversos analistas que han establecido los fundamentos de este enfoque y cuyos aportes utilizaremos selectivamente.

El marco conceptual sugerido por Michel Foucault acerca de los tres criterios para la "individualización" de un discurso nos proporcionará un esquema para la descripción del discurso sobre la corrupción. Foucault identifica las reglas de:

Corrupción y desarrollo: Deconstruyendo el discurso del Banco Mundial 
1. Formación: para todos sus objetos, conceptos y opciones teóricas.

2. Transformación: las condiciones que deben haber sido efectivas en el momento preciso para que [el discurso] fuese formado; qué modificaciones internas fue capaz de; desde que umbral de transformación las nuevas reglas han sido puestas en juego, y

3. Correlación situada entre otros tipos de discurso y en el contexto no discursivo en el cual funciona (instituciones, relaciones sociales, la coyuntura económica y política)" (Foucault 1978: 9f).

El proceso de transformación es explorado en la tercera sección al discutir los cambios políticos, económicos e ideológicos localizados paralelamente al viraje realizado por el discurso del BM. La cuarta sección intenta desmantelar una selección de reglas de formación, expresadas como dicotomías fundamentales de categorización de información, relaciones sociales, motivación del actor y los medios de intervención. La correlación de otros discursos (especialmente 'el estado', 'la burocracia' y el paradigma neoliberal) y el contexto no discursivo (por ejemplo, el BM como institución) será discutida en varias ocasiones en el texto.

Arturo Escobar (1995) ha adaptado los planteamientos de Foucault al estudio de los aspectos discursivos del desarrollo y ha analizado cómo el "Tercer Mundo" ha sido producido por el discurso del "desarrollo" después de finalizada la Segunda Guerra Mundial. Así, de acuerdo con Escobar, la emergencia del paradigma modernista del desarrollo engendró la profesionalización e institucionalización del campo y "creó un aparato extremadamente eficiente para producir un conocimiento acerca de éste y ejercer el poder sobre el Tercer Mundo" (1995: 9). Siguiendo este argumento, se puede afirmar que la concepción que tiene el BM de la corrupción es localizable dentro de este contexto, y se constituye en una continuación directa de la producción y control del conocimiento, así como sobre los resultados deseados del desarrollo.

La concepción de Escobar en relación de la formación de discursos, sin embargo, es demasiado estática y unidireccional. El antropólogo colombiano divide el espacio del discurso en dos campos de actores (los impulsadores del desarrollo, aquellos que son impactados por el desarrollo y aquellos que resisten el desarrollo) que son construidos 
principalmente como homogéneos. A pesar de que los temas de resistencia y coexistencia de comprensiones diferentes son importantes, la variación dentro de cada "campo" y de su contínua interacción reflexiva no debe ser exagerada (Alfaro 2000).

D. B. Moore, al igual que Escobar, sugiere que el BM se encuentra en una singular posición de "sustentador de la ortodoxia", lo cual crea un consenso hegemónico a través de un proceso de cooptación de los contradiscursos radicales, tales como "sustentabilidad" y "buena gobernabilidad” (Moore 1995: 16). Este proceso de incorporación es central en el presente análisis.

Sin embargo, el tratamiento que se hace del BM como un actor hegemónico no capta muchos aspectos en su encuentro con el discurso sobre la corrupción. En primer lugar, como Foucault lo reconoció, no existe oposición entre los discursos sin vida y los sujetos todo poderosos que los manipulan; en cambio "los sujetos dicursivos son parte del campo del discurso" y así son influenciados por éste y al mismo tiempo lo influencian (Foucault 1978: 13). El BM también ha experimentado cambios debido a su adopción de la campaña anti-corrupción. En segundo lugar, no deben desestimarse los conflictos internos dentro del BM, por ejemplo el conflicto entre el departamento jurídico, el departamento de investigación y las oficinas regionales o la manera cómo reacciona institucionalmente ante los casos de corrupción. Tercero, el asumir su condición hegemónica, privilegia al BM como un agente proactivo y consciente y por lo tanto se deja a un lado la teorización de su papel como sujeto reactivo definido por presiones externas - tales como cambiar las prioridades de los donantes y clientes y la opinión pública. Cuarto, es crucial reconocer que los discursos nunca son simplemente impuestos por un grupo dominante a un subordinado. Las ideas dentro de el discurso del desarrollo pasa de un sistema internamente heterógeneo de conocimiento a otro sistema internamente heterógeneo a través de la economía política del desarrollo, de sus prácticas y de sus intervenciones. A través de este proceso social de migración de conceptos, las ideas se transforman y adaptan a un sistema de conocimiento particularmente histórico y espacialmente localizado. En nuestro caso es importante señalar que los discursos del BM sobre la corrupción no son aceptados en su totalidad por los gobiernos clientes, sino que son selectivamente y estratégicamente integrados en las concepciones y prácticas de la economía política de sus respectivos países. Esto implica reconocer la amplia variedad de 
gobiernos existentes en el Sur, los intereses específicos que éstos defienden y su posicionamiento internacional.

Finalmente, para contrarrestar el argumento hegemónico, se puede expresar que el BM se encuentra institucionalmente en riesgo por haber adoptado el tema de la corrupción. Esto es reconocido por algunos actores al interior del BM, especialmente en su departamento jurídico, el cual visualiza la amenaza que este problema plantea para la credibilidad del BM. Lo anterior hace extremadamente difícil mantener la diferencia entre las esferas políticas y económicas y plantea algunas interrogantes sobre la soberanía. El combate de la corrupción necesita efectivamente una acción al nivel de la gestión micro-económica; sin embargo, el BM no tiene ni el mandato ni la capacidad profesional para actuar efectivamente. El intento de definir la corrupción de un modo limitado, y colocar la responsabilidad final de su reducción en los gobiernos clientes podría por lo tanto ser interpretado como una movida defensiva para autoprotegerse de las expectativas y demandas agregadas.

Existen pocos trabajos que examinan la corrupción desde un punto de vista discursivo. Talvez se deba a su reciente introducción en el discurso dominante del desarrollo, esto es, si lo comparamos con nociones establecidas como "sustentabilidad", "participación" o "ayuda" que ya forman parte de la ortodoxia del discurso del desarrollo. Sin embargo, existe otro factor que distingue la "corrupción" de otros "objetos" discursivos y que hace su decontrucción políticamente difícil. La mayoría de los conceptos utilizados en el discurso del desarrollo son positivos: igualdad, colaboración, participación, progreso, sólo para mencionar algunos ejemplos tomados del Development Dictionary (Sachs 1992). La corrupción es un concepto negativo, algo que uno no desea lograr, sino combatir. De manera que llevar a cabo un ejercicio analítico de los programas de anti-corrupción del $\mathrm{BM}$ nos permitirá identificar las limitaciones, los sesgos e inclinaciones del discurso. De hecho, el presentar las críticas a la cruzada anticorrupción como antidesarrollo es una parte integral del discurso, y por lo mismo debe ser objeto de cuestionamiento.

\section{La emergencia del discurso}

En esta sección se establecerá el contexto y las coordenadas de la emergencia del discurso del BM en torno a la corrupción. En primer lugar se proporcionará un panorama de los cambios en la economía 
internacional y los correspondientes virajes en la teoría del desarrollo desde 1945. Luego se procederá a discutir los antecedents inmediatos del discurso sobre la corrupción que pueden encontrarse en los cambios económico-politicos e ideológicos que han ocurrido desde la finalización de la Guerra Fría.

Siguiendo a Foucault, el propósito de trazar la genealogía de un discurso es "demostrar su precariedad [y] hacer visible no su arbitrariedad, sino su compleja interconexión con una multiplicidad de procesos históricos". De esta manera es legítimo desafiar la "falsa autoevidencia"(Foucault 1981: 15) de que la campaña anti-corrupción del BM sea algo natural e indispensable. El enfoque genealógico se encuentra en claro contraste con la preferencia del BM por la "breve historia"; al respecto, la "historia oficial" retoma una serie de estudios e informes seguidos por las acciones que han realizado diferentes departamentos del BM; este relato da la impresión que los cambios en la política del BM son el resultado de una reacción natural al aumento de la cantidad de evidencia existente y de igual manera presenta, además, los cambios socio-políticos como irrelevantes.

Algunos antecedentes económicos, políticos e ideológicos vale la pena contextualizarlos. Así, después de finalizada la Segunda Guerra Mundial, la reestructuración de la economía internacional se basó en la creencia keynesiana de que el Estado podría superar las fallas del mercado. Este paradigma de intervencionismo estatal de las décadas del cincuenta y sesenta llevó a un período de un crecimiento económico mundial realmente excepcional y creó un aumento en las expectativas acerca del papel del estado, especialmente en el mundo periférico.

A principios de los setenta, la era del crecimiento vía la participación del estado llegó a su final y fue reemplazada por la creencia en el poder del mercado, la cual fue complementada con una marcada desconfianza hacia el estado (Gilpin 1995). Este paradigma neoliberal fue construido alrededor de un modelo de "búsqueda de la renta", un concepto que cubre, pero no es sinónimo, con la corrupción en el sector público. La renta, como la define Gallagher, es "el uso directo o el desperdicio de recursos económicos para obtener beneficios no económicos" (1991: 31) y las teorías de la "búsqueda de la renta" "fueron empleadas en su momento para mostrar que dadas las preferencias, los gobiernos de los países en desarrollo pueden servirse solamente a si mismos y a su (mayoría) de votantes urbanos" (Schmitz 1995: 64). Por lo 
tanto, en el interés de la equidad, se argumentó que el desarrollo debería basarse en el mercado y no en el estado. Esta concepción, con menores variantes, todavía es la dominante en la actualidad y muestra cuan profundos son los presupuestos que acerca de la corrupción y su vinculación con el estado sustenta la ideología neoliberal del BM.

Haciendo eco a la ideología neoliberal, ha existido una tendencia hacia la despolitización y tecnicalización del discurso del desarrollo (Ferguson 1990). Este escenario ha sido moldeado por el punto de vista prevaleciente en los países del centro, especialmente desde el fin de la Guerra Fría, en la que se afirma que las principales causales de los conflictos políticos han sido resueltos -a través de una gestión económica capitalista y con la democracia liberal- y que la única tarea aún pendiente es la gestión de estos logros en todas las regiones de la economía-mundo capitalista. Este proceso de despolitización y tecnicalización no constituye en sí mismo un proceso a-político, como Ferguson lo indica, sino que inherentemente involucra desigualdades de poder en la determinación de la agenda del desarrollo. El enfoque del BM -que persigue "mejorar" las instituciones del estado para reducir la corrupción- se origina en su pretensión de ser el genuino representante de los medios y las metas del desarrollo.

Vale la pena indicar que exisiteron antes de los noventa intentos de colocar la corrupción en la agenda internacional. En 1975, la Asamblea General de las Naciones Unidas propuso una resolución sugiriendo "medidas contra las prácticas corruptas de las corporaciones transnacionales". En 1979 ECOSOC sugirió un "código de conducta" y un "acuerdo internacional" sobre los "pagos ilícitos". Estas iniciativas encontraron una fuerte resistencia, tanto de los países de donde las empresas eran originarias como de los países que las hospedaban. Cuando los documentos fueron discutidos en la Asamblea General en diciembre de 1980, éstos fueron rechazados. Ahora la interrogante a plantearse es qué cambios hicieron posible que el tema fuera aceptable?

El fin de la Guerra Fría y los cambios que lo acompañaron en las relaciones Norte-Sur proporcionaron una oportunidad política de repensar las concepciones del "desarrollo" y evaluar los logros que hasta ese momento se habían materializado. Quedó establecido que la mayor parte de países de la periferia se habían beneficiado muy poco de cuarenta y cinco años de ayuda al desarrollo en un contexto de confrontación ideológica, y se plantearon interrogantes en relación del 
por qué si este era el caso, los enfoques teóricos dominantes (especialmente, el discurso ortodoxo neo-clásico) no fueron capaces de explicar dicho fenómeno.

La ortodoxia neoliberal fue puesta bajo presión dado que los programas de ajuste estructural impulsados por el BM, cuya base eran las recetas neoliberales, fueron fuertemente criticadas por organizaciones como la UNICEF y el PNUD. Además, a los desafios que enfrentaban los soportes teóricos del BM, las prioridades de sus miembros también experimentaron cambios. Los miembros donantes fueron adaptando sus prioridades de ayuda a ajustados presupuestos nacionales y a la opinion pública doméstica - hubo una demanda de una mayor fiscalización sobre los resultados del desarrollo y el apoyo a dictadores corruptos basado en consideraciones estratégicas ya no era aceptado. El crecimiento de los movimientos sociales democráticos en los países de la periferia hizo eco de este sentimiento. Los donantes decidieron que la ayuda debería limitarse a gobiernos "confiables" (Schimtz 1995: 70).

De todas estas preocupaciones y presiones, nació el paradigma de la "buena gobernabilidad" (good governance). Se puede observar que dicho paradigma se constituyó en el precursor necesario del compromiso abierto adoptado por el BM en el tratamiento de la corrupción. "La Gobernabilidad" en los paises en desarrollo (no globalmente o en el Norte)" fue identificada tanto por el BM como por los países donantes de la Organización para la Cooperación Económica y el Desarrollo (OECD) "como la fuente primaria del problema (explicando la falta de logros de la benevolencia pasada), y como la base para la solución (justificando nuevas condiciones y limitaciones sobre esta benevolencia)"(Schmitz 1995: 68). Schmitz interpreta la adopción del modelo de la "buena gobernabilidad" como una estrategia defensiva para prevenir una crisis percibida del paradigma neoliberal a través de la cooptación del discurso crítico. En lugar de ser un movimiento transformador y progresista, Schmitz argumenta, el paradigma neoliberal fue finalmente protegido mediante cambios en los centros de atención: de factores sistémicos internacionales como "las condiciones adversas, mercados injustos o reformas económicas inapropiadas" a factores asociados con problemas locales como "la ausencia de una capacidad institucional para administrar los procesos de ajuste necesarios” (Schmitz 1995: 76-68). Más adelante, el Banco Mundial y los donantes de la OECD acordaron "afianzar los condicionantes políticos dentro del régimen de la buena 
gobernabilidad a una condicionante económica ortodoxa y a los fundamentos del desarrollo "amigables al mercado"(Schmitz 1995: 71). Esta vinculación es expresada muy claramente en algunos de los programas anti-corrupción del BM (Gray y Kauffman 1998: 29).

No queda del todo claro que la discusión abierta sobre la corrupción se haya nutrido del enfoque de la "buena gobernabilidad". Existieron problemas de inconsistencia conceptual interna y de inercia institucional. La introducción de la corrupción como centro de atención en el debate de la "buena gobernabilidad" es, por un lado, un paso lógico en la tendencia neoliberal del despolitizar el aparato de estado para lograr un avance en los mecanismos de mercado, y por otro, el debut de la corrupción en 1996 en el Banco concincidió con el Informe del Desarrollo Mundial, con el cual se enfrenta conceptualmente.

El momento decisivo para la utilización abierta del término corrupción ocurrió en la Reunión Anual del BM y del FMI en 1996, cuando el presidente del BM James Wolfensonhn expresó un compromiso público de combatir la corrupción. Esto proporcionó el impulso interno para desarrollar un esquema de acción de combate a la corrupción por parte del BM.

\section{Deconstruyendo el discurso}

Antes de discutir los cinco conjuntos de dicotomías que apuntalan la concepción del BM sobre la corrupción, nos detendremos en comentar la lógica de las reglas de formación del discurso. Las estrategias utilizadas para escoger la definición de corrupción, argumentamos, sirve para examinar a través de un "complejo y sensitivo" (World Bank 1997b) conjunto de relaciones y experiencias que han servido para seleccionar, y activamente construir, aquellos aspectos que no contradicen las autoconcepciones que existen en el BM.

Un texto fundamental del BM que examina la corrupción, implicitamente reconoce que una concepción muy particular de corrupción fue escogida por ser consistente con las categorías de pensamiento y de la praxis del BM. "Al considerar su estrategia, el Banco buscó una definición utilizable de corrupción y luego desarrolló una taxonomía de las diferentes formas de corrupción que podría ser consistente con esa definición". La definición de conceptos de acuerdo a las necesidades de una organización es angustiosamente común en la "industria del 
desarrollo". El peligro de la tautología y de los hallazgos de lo que uno espera encontrar debe quedar claro.

Podemos describir el proceso mediante el cual la corrupción ha sido incorporada en el discurso existente del BM de dos maneras: la primera, que ésta se ha convertido en un "concepto"operativo de acuerdo a ciertas reglas, y la segunda, que ha experimentado un proceso de "desmistificación".

Una vez que algo se convierte en "concepto", se le da características y parámetros fijos: se convierte en un objeto de estudio, de política y de intervención. Mientras la corrupción fue discutida en el mundo académico y en los círculos ajenos al BM antes de 1996, ésta no tenía las caracterísiticas centrales que le permitieran ser reconocida como un "concepto operativo" por el BM: era demasiado política. Solamente cuando se hizo la transición hacia un "concepto económico" pudo comenzar el proyecto de definir, medir y encontrar las causas y los efectos del problema identificado. Una vez fue aceptada, todo un universo de experiencia e información se convirtió en relevante y "expresable".

La incorporación del término "corrupción" dentro del rango de conceptos aceptables por el BM fue seguido de un proceso que incorporó dos pasos de "desmistificación". Primero, factores institucionales de "gobernabilidad" tuvieron que ser reconocidos como relevantes para la práctica del $\mathrm{BM}$ de manera que expandiera su campo de "intervención”. Este proceso incluyó la reconceptualización de categorías políticas para que fueran coherentes con las formas económicas de entendimiento y expresión. Un segundo paso consistió en definir que la noción real de "corrupción" podría ser relevada de su estatus de "tabú" porque podría ser controlada al ubicarla dentro de la novedosa y ampliada esfera económica.

La primera etapa es ejemplicada en los hallazgos encontrados por Robert Klitgaards cuando en 1989 fue invitado a entrevistar a personal del BM acerca de lo que la institución podría y debía hacer acerca de "los fenómenos agrupados bajo la etiqueta de "corrupción". Klitgaards resumió el sentimiento que encontró entre los empleados que entrevistó de la siguiente manera:

“1. Evitar la palabra corrupción, enfatizando en su lugar en aspectos como la eficiencia administrativa, desarrollo institucional o el ajuste structural del mismo gobierno

Corrupción y desarrollo: Deconstruyendo el discurso del Banco Mundial 
2. Las sensibilidades serían suavizadas al identificarse maneras prácticas y trabajables para reducir la corrupción" (citado por Tesh 1999: 2).

El pasaje ilustra dos puntos. Uno es que la división entre las esferas políticas y económicas parecen ser definidas con base a la aplicabilidad de cierto enfoque intervencionista con relación a la esfera política, no considerando, en tal sentido, otras características. Así, "administraciones", "instituciones" e incluso "gobiernos" se convierten en no-políticos y por lo tanto abiertos a intervenciones externas tan pronto como son conectados con "eficiencia", "desarrollo", y especialmente con "ajuste structural". El segundo punto, es que la definición del concepto de corrupción se hace dependiente de la percepción de las posibles intervenciones, en lugar de adaptar el rango de intervenciones a un amplio análisis del problema (Ferguson 1994: 177).

Podemos identificar tres aspectos más del discurso. Estos tienen el efecto de minimizar los aspectos disonantes que existen en las autoconcepciones existentes en el BM. Primero, y fundamentalmente, corrupción como palabra es una herramienta "ajena". No describe una acción específica (por ejemplo, robo) sino es un concepto de evaluación de carácter negativo que nos dice poco acerca del comportamiento en sí mismo (una transferencia de recursos) que acerca del sistema de valores de la persona o sociedad que ha sido señalada. Euben hace notar que "llamar a un régimen corrupto es decir algo acerca de las preferencias del que expresa el juicio, pero no del régimen mismo"(1989: 230).

Un segundo mecanismo, para controlar el discurso sobre la "corrupción”, es la construcción de un consenso con respecto a qué clase de información podría legítimamente contribuir a definir el camino hacia la "verdad" respecto a la corrupción. Foucault describe esto como una característica central de un discurso: "[cuando me refiero] a la producción de la verdad, quiero decir no a la producción de la verdad absoluta sino al establecimiento de dominios en los cuales la práctica de lo verdadero y lo falso puede ser hecha de una vez en forma ordenada y pertinente" (1981: 9). Las esferas del conocimiento aceptadas por el $\mathrm{BM}$ son universalistas, empíricas, cuantitativas, institucionales y basadas en la concepción burguesa (moderna) de la actitud calculadora y racional del sujeto que busca maximizar su individualidad. Los puntos de vista alternativos (por ejemplo, las comprensiones morales, culturales o políticas sobre la corrupción) son considerados como argumentos ingenuos, especieros o maliciosos realizados por partes interesadas (por 
ejemplo, empresarios o políticos corruptos). Gadbaw y Richards reflejan este consenso manufacturado cuando puntualizan: "de país a país, en todas las regiones y entre todas las culturas del mundo, este cinismo [de relativismo cultural al definir la corrupción] parece haber sido barrido" por la abundante "evidencia" sobre la "magnitud y los alcances" del "problema" (1997: 3).

Tercero, la variación en las concepciones y experiencias de la corrupción en diferentes países, al mismo tiempo que se les reconoce, son consideradas solamente como adición a ciertas características centrales y fijas de la corrupción. De acuerdo a Schmitz, "el mensaje ingenuo reduce la confianza en la benevolencia y en el conocimiento superior de Occidente. Entonces, y sólo entonces, uno puede permitirse hacer las adaptaciones apropiadas para las condiciones locales" (1995: 59).

A pesar de las estrategias empleadas para hacer "seguro" el discurso para el BM, el proceso de adopción y adaptación de un discurso es conflictivo: las percepciones sobre corrupción no son homogéneas en las diferentes instancias del $\mathrm{BM}$ y el proceso de adaptación no es unidireccional. Como se ha indicado anteriormente, el discurso incluso podría poner en riesgo la práctica y el auto-entendimiento del BM. Sin embargo, existen cinco categorías dicotómicas que centralizan la estructura de las concepciones del BM sobre corrupción que se discuten a continuación.

\section{Crisis/Continuidad}

El discurso del BM sobre corrupción ha debido tartar cuidadosamente con dos niveles de cambio y continuidad. Uno es cómo analizar la crisis y la continuidad en sus sujetos (por ejemplo, en Centroamérica), y el otro es cómo se construye el proceso de cambio y continuidad de respuesta al interior de la organización. Desde nuestro punto de vista, ambos son presentados de modo que los temores de un cambio radical en la política del BM, ya sea dentro o fuera de la institución, son tranquilizados; al mismo tiempo que las actividades reales son expandidas sustancialmente.

Es muy común encontrar en los textos de políticas de desarrollo la construcción de un conjunto de problemas, idealmente en condiciones de crisis (Berstein 1990: 5), para justificar la intervención. Es interesante observar que el BM no hace esto para justificar su interven- 
ción en el nuevo campo de la corrupción. En el documento de enfoque de 1997 no existe ninguna mención sobre un rápido incremento en las prácticas de corrupción o de la crisis; en su lugar las "causas de la corrupción" son presentadas como modelos estáticos y ahistóricos (World Bank 1997b). La novedad que justifica la intervención es presentada como un aumento en la demanda externa a la cual el BM está respondiendo: "la corrupción es una preocupación creciente para los donantes, organizaciones no gubernamentales y gobiernos y ciudadanos en los países en desarrollo y en los industrializados, una pequeña pero creciente cantidad de países se ha acercado al BM solicitando asistencia" (World Bank 1997b). La construcción de la demanda externa sirve para un doble propósito: hacer el "nuevo" centro de atención menos amenazante y absolver al BM por no haber considerado antes el mencionado "gran obstáculo al desarrollo equitativo y sustentable".

Los factores históricos que hicieron que la demanda apareciera en el momento actual, y que le permitieron al BM responderla, no se mencionan. Tampoco existe una perspectiva histórica de las causas que han hecho emerger la corrupción en los países de la periferia. Esto es parte de la construcción de la corrupción como un aspecto externo al BM, y al colonialismo, descolonización, la deuda y la economía internacional. La concepción del $\mathrm{BM}$ de la economía internacional como contribuyente a los efectos del "desarrollo" negativo está fuera de las fronteras "expresables" del discurso (Foucault 1978: 14-15), ya que podría poner en peligro el proyecto de una intervención continuada, expandida y orientada por las directrices neoliberales (Bourdieu 1998).

La estrategia de justificar la intervención solo a través de las demandas se enfrenta, sin embargo, con la necesidad de mostrar el alcance del daño causado por la corrupción. Gray y Kaufman indican que en una encuesta de 150 funcionarios públicos de alto rango de 60 países, los entrevistados establecieron el incrmento de la corrupción en el sector público como "el impedimento más severo para el desarrollo, así como para el crecimiento en sus países" (1998: 22). Esto podría indicar una verdadera crisis, lo cual obliga a preguntarse, por qué los funcionarios entrevistados no lo habían identificado antes, y por qué tampoco por el $\mathrm{BM}$, que es la institución que realizó la encuesta?

En la presentación del documento de enfoque del BM, en donde se introduce por primera vez el "nuevo" tema, el balance entre lo novedoso y la continuidad en las respuestas del BM a la corrupción está 
también orientado hacia la continuidad. "El Banco hace bastante tiempo ha estado preocupado por controlar el fraude y la corrupción en sus proyectos, el Banco se encuentra apoyando la política y la reforma institucional que, entre otras cosas, contribuya a la lucha contra la corrupción" (World Bank 1997a). Los programas de ajuste structural, afirma el BM, al "reducir las rentas económicas" ha contribuido a reducir las oportunidades para generación de casos de corrupción (World Bank 1997a). Esta construcción de continuidad es similar a la forma mediante la cual el enfoque de la "gobernabilidad" fue presentado en el informe Gobernabilidad y desarrollo, de 1992. En dicho Informe se lee: "no hay necesidad de criterios adicionales para reflejar las preocupaciones con la gobernabilidad: simplemente la aplicación efectiva y consistente de los criterios existentes" (World Bank 1991: 19).

A pesar de esta retórica, ambas voces dentro y fuera del BM interpretaron la "preocupación con la gobernabilidad", como un rompimiento significativo con los parámetros previos. El departamente jurídico fue muy precavido en no pasarse de la raya en los requerimientos del BM de mantenerse "apolítico" (World Bank 1995). De igual manera, existe una relación no confusa entre la retórica y la continuidad, y su verdadero alcance con respecto a los programas anti-corrupción.

Finalmente, si el BM ha venido reduciendo las oportunidades para se generen casos de corrupción con el lanzamiento de los programas de ajuste desde la década de los setenta, ¿por qué la corrupción ha persistido o incluso se ha incrementado significativamente? El BM no analiza cómo la continuidad de sus propias acciones del pasado al presente ha podido contribuir a estimular la corrupción. Algunos préstamos iniciales en el marco de los programs de ajuste, realizados con un alto nivel de discresión, por ejemplo, son considerados responsables de estimular enormes niveles de corrupción de parte de los gobiernos destinatarios de dichos fondos. La contribución del BM en el debilitamiento y desligitimación de gobiernos, y por lo tanto de un agente que ha estimulado la corrupción, se discute en la siguiente sección.

\section{Lo político/Lo económico}

"Corrupción" era considerada una noción "tabú" porque era definida arbitriariamente como un concepto estrictamente "político". La exclusion de lo "político" del ámbito del BM está inscrito en sus artículos de acuerdo (World Bank 1990). La separación de lo económico 
y lo politico fue introducido porque el $\mathrm{BM}$ es una institución multinacional; sus miembros son formalmente estados, y en la práctica gobiernos, que no quieren que sus decisiones se vean influenciadas o limitadas. Así, "en el pasado el Banco con frecuencia era renuente a enfrentar la corrupción abiertamente debido a problemas de sensibilidad política y por la falta de demandas concretas para asistir a los clientes en esta área" (World Bank 1997b: 12). Ahora, sin embargo, "corrupción" ha sido definido como un concepto económico cuyas causas, "instituciones débiles y políticas gubernamentales específicas" (World Bank 1997b: 12), tales como regulaciones, pueden ser solventadas con la ejecución de "reformas económicas e institucionales" (Gray y Kaufmann 1998: 21).

No solamente "lo político" es excluido de una consideración efectiva; también es construida como inferior al punto de vista económico. El BM ha desarrollado una limitada y antogónica concepción de la política, lo cual se refleja en su tratamiento del problema de la corrupción. Como una ilustración a la afirmación anterior, discutiremos las construcciones del BM con relación al (1) papel del estado; (2) mundo de la "política"; (3) papel de la "voluntad política" y las decisiones de los gobiernos y (4) la relación de la "información" con la política.

\section{El papel del Estado}

El BM construye analíticamente al estado básicamente como proveedor de servicios para la economía y no como una entidad política cuya legitimidad se deriva de su creación por la ciudadanía y de su responsabilidad ante ella. En el World Development Report de 1997 se establece claramente que el concepto de legitimidad ha sido transfigurado en un problema técnico de credibilidad, especialmente cuando se trata de los inversionistas internacionales (Munro et al: 1999: 82). Lo que la mayoría de gobiernos, especialmente de aquellos países con estados débiles, están principalmente preocupados es no servir al mercado sino en fortalecer su base de apoyo, ya sea a través de la transferencia de recursos o con el establecimiento de medidas normativas de legitimidad. Para ambas estrategias, corrupción o su condenación son vitales. Debido a su comprensión simplista del estado, el BM no toma en cuenta tales usos políticos de la corrupción y la lucha contra los mismos como un factor constitutivo en la construcción del estado y la nación, si bien sus propios programas anti-corrupción son inevitable-

\section{2}

Realidad 102, 2004 
mente reinterpretados a la luz de las luchas políticas que se desarrollan en el estado-cliente.

\section{Lo "politico"}

El documento en donde el BM reconoce en principio que "la corrupción tiene una dimensión política y que refleja la manera en la que el poder es ejercido en un país" (World Bank 1997b: 5), tiene una definición de lo "político" bastante reducida. Está basada en una comprensión institucional de la distinción entre lo "burocrático" y la corrupción "política" de acuerdo al estatus formal de los actores: corrupción política, por lo tanto, involucra a parlamentarios y otros funcionarios públicos electos. Este "tipo" de corrupción es visto como un factor que va más allá de la jurisdicción formal del $\mathrm{BM}$. Al no aceptar una concepción de la política en sentido amplio (por ejemplo, las disputas sobre el matenimiento o cambio de las relaciones de poder) el BM cubre el hecho de que sus propias intervenciones en las actividades de los estados, las que define como puramente económicas, tienen también grandes efectos en las estructuras de poder de los mismos.

\section{El papel de la "voluntad política"}

Inclusive cuando el BM le brinda prioridad a la "voluntad política", la verdadera libertad de decisión ofrecida es altamente restringida. La separación artificial entre los actores locales que proporcionan la "voluntad política" y el BM, la cual "debe preocuparse únicamente con las causas y consequencias económicas" tiene dos efectos (World Bank 1997b: $5,24)$. En primer lugar, parece colocar la iniciativa y el poder de definición en el gobierno, el cual por su propia decision ha invitado al BM a asistirlo. A pesar que en apariencia este pueda ser el caso, el antecedente de una invitación para combatir la corrupción puede ser a menudo la necesidad que el gobierno tiene de créditos y la adopción paralela de la nueva retórica de moda. De cualquier modo, la libertad de definir los términos del compromiso es limitada, ya que la decisión consiste en aceptar o no un programa que coincida con la concepción anti-corrupción del $\mathrm{BM}$; es decir, la reforma económica neoliberal y la construcción de la capacidad institucional inspirada en el model weberiano del modelo burocrático. La aparente auto-restricción del BM de "aconsejar en aspectos relacionados con la reforma de la política económica y de fortalecer la capacidad institucional" oscurese el alto grado de 
control que ejerce sobre el contenido de las decisiones realizadas por los politicos. Mosley et al. (1995: xiii) enfatizan que el proceso de negociación está basado en políticas de préstamo y de condicionalidad, pero mientras (a un nivel formal) esto puede incluir, por ejemplo, la determinación de definir cual empresa tendría que ser privatizada como parte de un programa anti-corrupción, es improbable la posibilidad de modificar las receta de privatización, no digamos las bases económicas e institucionales en torno a las cuales descansa el enfoque que tiene el $\mathrm{BM}$ sobre las prácticas de corrupción.

En segundo lugar, la separación de las esferas política y económica tiene un efecto sobre la legitimidad de los gobiernos. El control sobre los procesos económicos es un instrumento fundamental que poseen los gobiernos para ganar y retener la legitimidad de ante sus ciudadanos. Este es especialmente el caso de aquellos países que basan su legitimidad en el ofrecimiento de promesas de bienestar social y donde el estado está todavía lejos de ser el actor económico más importante. Una vez que sea claro para población que las decisiones económicas no son tomadas libremente por sus gobiernos, sino por un actor externo, el compromiso con el estado puede deteriorarse. Así, las reformas basadas en la separación de las decisones económicas de las políticas puede incluso contribuir al aumento y/o aceptación de las prácticas de corrupción, y no de su combate.

\section{Lo público/ Lo privado y lo político/ Lo administrativo}

$\mathrm{Al}$ definir el espacio social en donde las prácticas de corrupción parecen llevarse a cabo, la distinción entre política y economía está estrechamente conectada a la formulación de esferas separadas para el estado y la sociedad (lo público y lo privado) y al interior del estado (lo político y lo administrativo). Ambas distinciones, están basadas en construcciones weberianas de "tipos ideales", las cuales han sido elaboradas a partir de experiencias típicamente europeas y que incluso no describen en forma precisa las relaciones en el "Occidente" capitalista. Esta posición contrasta con los argumentos simplistas del BM acerca de que sus reformas administrativas, y las medidas de fortalecimiento institucional que impulsa, están basadas en una imágen universalmente aplicable del estado y de las relaciones Estado-sociedad. Estos argumentos son los que inspiran el diseño de los programas anticorrupción, ya que ellos proporcionan el modelo implícito contra el cual el "disfuncional"estado corrupto es medido y evaluado. 


\section{Lo público/Lo privado}

La concepción de corrupción que prevalece en el centro occidental del sistema-mundo capitalista, en la cual el BM se inspira, depende de la existencia de un dominio público que se reconoce separado de la esfera privada, con diferentes códigos de conducta aceptables para cada uno. Mientras en el sector privado, se espera que la actividad de compañías e individuos se oriente hacia la búsqueda de ganancias y al enriquecimiento, organizaciones e individuos en el sector público se espera, por el contrario, que actuen desinteresadamente en función del bienestar público. Una acción corrupta es aquella que contravenga este modelo. Akhil Gupta desde una perspectiva antropológica argumenta que esta distinción entre lo público y lo privado está lejos de tener un carácter universal y que es predicada tomando únicamente la experiencia histórica de Europa (1995: 376). Como lo plantea Theobald, "tanto la idea como la realidad de la oficina pública son procesos de desarrollos históricos recientes. En tiempos premodernos, la supuesta importancia de la distinción entre las esferas públicas y privadas no existía" (1990: 4).

\section{Lo político/Lo administrativo}

El contraste percibido entre lo político y lo administrativo es también crucial en la interpretación que el $\mathrm{BM}$ hace de las prácticas de corrupción. Las concepciones eurocéntricas de una administración nopolítica se basan en una lectura simplista de la descripción de los "tipos ideales" que Weber realiza de una burocracia racional-legal. Desde este punto de vista un "servidor público de carrera, supuestamente, hace sus decisiones tomando como base criterios neutrales y universales, y escrupulosamente separa los asuntos públicos de los intereses personales" (Theobald 1990: 3). En definitiva, el BM afirma: "La corrupción se opone a los valores burocráticos de equidad, eficiencia, transparencia y honestidad" (World Bank 1997b: 17).

Esta concepción de burocracia es intrinsecamente normativa. Aunque el BM claramente señala cuales son los problemas con los sistemas y las sociedades sujetas a revision, raramente existe una afirmación clara de como tendría que ser la adminsitración ideal. Cualquier formulación positiva está basada en palabras ambiguas y cargadas de juicios de valor como "el servicio civil profesional, un manejo financiero adecuado, un proceso disciplinado de elaboración de políticas" (World 
Bank 1997b: 39). Esta imágen ideal de las características de una burocracia no está basada en un análisis real de cómo las burocracias industrializadas trabajan; sin embargo, sirve para "proporcionar de manera implícita una base para contrastarlas con las burocracias del Tercer Mundo, las cuales supuestamente están plagadas de favoritismo, faccionalismo y personalismo, y que de igual manera están altamente politizadas" (Theobald 1990: 13).

Además, el BM abstrae las actividades administrativas de cualquier importancia política que las mismas puedan tener. "La preocupación aquí no es con el ejercicio de los poderes del estado en el sentido amplio, sino específicamente con el manejo apropiado del sector público y la creación de un ambiente adecuado para el sector privado" (World Bank 1997b: 24). Este pasaje sugiere que es posible separar la técnicas de la buena gestión ("una preocupación por las reglas que son actualmente aplicadas a las instituciones que aseguran la aplicación apropiada de éstas" [World Bank 1997b: 24]) del contenido de lo que es gestionado. Todavía más, no hay un reconocimiento que la existencia, amplitud y actividad de la burocracia puedan tener un significado político.

Un problema final con el argumento que asume la estricta división de lo "político" y lo "administrativo" es que —analizándolo desde una perspectiva institucional - no describe los verdaderos procesos que se desarrollan en la toma de decisiones. Como la teoría micro-institucional lo indica, "las decisiones burocráticas no son neutrales e imparciales sino también son influenciadas por consideraciones individuales de poder, ganancia e interés" (Theobald 1990: 13).

Sería equivocado afirmar que el BM intenta imponer una concepción única y estatica del "estado correcto" en el mundo, principalmente porque su propia concepción ha cambiado significativamente con el tiempo. En el espacio de unos años en la década de los noventa, el BM cambió de una posición que defendía que "el no gobierno es el mejor gobierno" a una que apoyaba "el tamaño correcto (sic) de un gobierno" (World Bank 1997c). De todos modos, el distinguir entre "el estado" y la sociedad, así como el contrastar los valores y la práctica de lo político y de lo adminstrativo, tiene raíces profundas y fundamentalmente afecta la manera cómo los niveles de corrupción son medidos y cómo los programas anticorrupción son diseñados. 


\section{Lo individual/ Lo institucional y lo moral/racional}

Otra característica central del discurso del BM sobre el tema es su tendencia a prestar particular atención a las estructuras institucionales más que en el actor individual. El individuo corrupto es construido como un maximizador racional que responde a incentivos, en lugar de concebirlo como un agente moral. Por lo tanto, se enfatiza en la reforma institucional, para prevenir futuras prácticas de corrupción, lo cual está en oposición a perseguir a los individuos responsables de actos de corrupción en el pasado. Además, las instituciones son reformadas evocando criterios racionales de eficiencia y sin considerar un modelo moral de la "polis buena".

Las concepciones morales son específicamente rechazadas por el $\mathrm{BM}$, ya que la "condenación" se visualiza como un impedimento para hacer un "análisis objetivo". Como se ha mostrado con anterioridad, incluso la afirmación de que se trata de elaborar un análisis "objetivo", contiene un elemento normativo. Además, el dominio de una concepción amoral, es decir, racional de la corrupción es muy reciente y no tiene una carácter universal, incluso en la actualidad. Las concepciones religiosas sobre la corrupción que todavía tienen una gran influencia en la retórica política norteamericana, y en el mundo islámico ortodoxo es concebida como un pecado. El problema es qué función tiene el discurso institucional. Además de ser coherente con la vision racional, secular y modernista presente en el enfoque del BM, éste es un medio estratégico para iniciar el cambio a un nivel que sea favorable a la intervención externa; también esta visión evita abiertamente desafiar el status quo de los individuos o grupos que se encuentran en el poder.

\section{Política/ implementación y proceso/ resultado}

Muchas de las dicotomías indicadas que constituyen la base del enfoque del BM sobre corrupción, directamente afectan la concepción del proceso mediante el cual sus programas anti-corrupción son llevados a cabo. Dos aspectos estrechamente relacionados serán discutidos a continuación. Primero, el BM trabaja con un modelo implícito de política e implementación que es un clásico ejemplo de lo que Bernard Schaffer llama el "la falacia de la política del sentido común". Este modelo asume que la política es una "actividad voluntarista, temática de varios actores [quienes] son vistos como outsiders inocentes". La implementación es separada y "se realiza más tarde" (Shaffer 1984: 
142). Shaffer argumenta que este modelo no describe lo qué realmente pasa y no es apropiado para el logro de los mejores resultados, pero que sirve a los propósito de los elaboradores de política (en nuestro caso, los empleados del BM), ya que los absuelve de la responsabilidad de los fracasos que puedan surgir en el proceso de implementación. De acuerdo con este modelo, los elaboradores de política, no son responsables por tomar encuenta las dificultades reales en la implementación de sus ideas en los dispositivos contenidos en los planes. Las preocupaciones con la "buena gobernabilidad" y la corrupción puedrían ser vistas como un paso hacia la implementación de soluciones a los problemas que rodean las reformas de ajuste estructural impulsadas entusiastamente por varios años por el BM. Sin embargo, las metas parciales de una mejor gobernabilidad y menos corrupción no tienen probabilidad de ser logradas, porque los modelos limitados de gobernabilidad y corrupción utilizados en la formulación de las políticas son incapaces de capturar las complejidades más relevantes.

La separación en etapas de investigación, política e implementación es exacerbada, en el caso del $\mathrm{BM}$, porque es correlativa con una completa separación de los actores. El modelo dicta que la política debe ser elaborada por el BM, mientras los actores políticos nacionales son responsabilizados con la preparación e implementación de las reformas (Woods 2000: 825). Esta separación reduce la cantidad de coordinación entre las diferentes partes del proceso en su totalidad y permite a ambas partes de acusarse de errores en el trabajo en el caso de un proyecto fracasado. Como Shaffer lo hace notar, esto implica aprender de los errores del pasado y de esa manera intenta reducir "el espacio de maniobras hacia políticas alternativas o mejores" (2000: 827).

El segundo punto está vinculado con la manifestación consciente de las relaciones entre el proceso de la elaboración de políticas y de sus resultados, es decir, de la cantidad de control que se necesia para el logro de un programa. Nuestro argumento es que la comprensión que tiene $\mathrm{BM}$ de cómo tratar la corrupción es lógicamente contradictoria con la nueva ortodoxia basada en una maximización del control (Woods 2000: 824). La idea de desarrollar programas efectivos para reducir la corrupción es incompatible con la idea de un incremento en el control del gobierno ya que los mismos actores son simultáneamente construidos como los portadores del problema principal, y también de su solución. En sus inicios, la mayor parte de los préstamos dentro de los 
programas de ajuste estructural, fueron proporcionados permitiendo una alta discresión gubernamental en el manejo y destino de los fondos. Actualmente, esto es considerado como una de las causas que explican el incremento de la corrupción que se evidenció dramaticamente durante la década de los ochenta.

El BM argumenta que ha adoptado una concepción pluralista de la propiedad que se enfoca en la sociedad civil en lugar de los gobiernos, y que siempre hay personas reformadoras y departamentos menos corruptos al interior del estado. Sin embargo, esta celebración de la cooperación con las organizaciones de base es contrarrestada por un aumento en la tendencia hacia la elaboración de planes técnicos altamente complicados y que sirven de base para las relaciones que se establecen con los gobiernos clientes. Las reformas institucionales, que son parte de los programas de fortalecimiento gubernamental y de los paquetes anti-corrupción, requieren una burocracia eficiente que los ejecute, lo cual por definición es inexistente en las estructuras gubernamentales de los países clientes.

En el mediano plazo, el BM tiene que percibirse como una entidad que actúa sobre problemas que se han convertido en significativos en la palestra pública del desarrollo. Por lo tanto, acitividades y textos se constituyen en necesarios para responder a las críticas que señalan que el BM no está haciendo lo suficiente para combatir las prácticas de corrupción. En el largo plazo, la responsabilidad (no realista) de los gobierrnos clientes absuelve al BM del (posible) fracaso. Uno podría incluso ver al BM como una "víctima" de los aspectos técnicos, pero también morales, del discurso del desarrollo que ha contribuido a construir.

\section{Conclusión}

Este trabajo ha presentado solamente las líneas generales del discurso del $\mathrm{BM}$ acerca de la corrupción. Este esfuerzo es una primera introducción a un enfoque que todavía está sin explorarse en toda su amplitud. La caracterización del discurso en téminos de dicotomías contribuye a descubrir las inconsistencias internas que necesitan ser reconocidas y discutidas. Sin embargo, las dicotomias no le prestan demasiada importancia a muchas de las sutilezas y pueden crear una imagen del BM como un organismo portador de una actitud que trasciende una mentalidad cerrada y monolítica. Existen una serie de patrones dentro del BM que definen cuales medios son considerados aceptables o inaceptables 
en la estructuración del conocimiento en torno a complejas realidades sociales; éstos han sido puestos en juego - como se ha tratado de demostrar - una vez más en el discurso sobre la corrupción. Estos patrones están estrechamente relacionados con la economía política de las estructuras internas y con las relaciones externas que la organización establece con los "clientes" donantes y con el público. Un análisis a profundidad de esta relación, o sea entre discurso e institución, aún falta realizarlo y es un desafio que la critica al discurso del desarrollo debe urgemente articular.

¿Qué efecto tendrá la adopción de este discurso particular en el "mundo en desarrollo"? Esta interrogante define el esfuerzo que se ha realizado para desconstruir el discurso de la corrupción dentro de la narrativa institucional del $\mathrm{BM}$; una respuesta categórica a la interrrogante planteada requiere análisis posteriores de las concepciones de corrupción que se utilizan actualmente a nivel mundial, las cuales interactúan diariamente con las intervenciones anti-corrupción promovidas por el BM. Corrupción puede ser otra "moda" para el BM y para la industria del desarrollo (Tesh 1999). Sin embargo, nuestro análisis sugiere que el discurso mismo está firmemente inscrito en el gran proyecto de modernización capitalista del $\mathrm{BM}$ y que en la realidad extiende su alcance al impactar las vidas y las mentes de las gentes en innumerables regiones del planeta. Lejos de ser el resultado de simples ajustes técnicos de un sistema que se encuentra en movimiento, el discurso de la corrupción categoriza y en tal sentido tiende a deslegitimar sociedades completas. "Una buena sociedad es aquella que se moderniza; una sociedad corrupta es aquella que que se inhibe al 'desarrollo"' (Euben 1989; 243).

\section{REFERENCIAS}

Alfaro. S.O. (2002) "Critical Remmarks on Development Discourses". Departmento de Sociologia y Estudios Sociales, Universidad de Regina (Seminario de Investigación).

Berstein, H. (1990) “Agricultural 'Modernisation' and the era of structural Adjustment: Observations on Sub-Saharan Africa", Journal of Peasant Studies 18(1).

Bourdieu, P. (1998) Acts of Resistance. Against the Tyranny of the Market. Nueva York: New York Press.

Derrida, J. (1978) Writing and Difference. Londres: Routledge and Kegan Paul. 
Eade, D. (ed.) (1997) Development and Patronage; a Development in Practice Reader. Oxford: Oxfam.

Escobar, A. (1995) Encountering Development: The Making and Unmaking of the Third World. Princeton: Princeton University Press.

(1997) "Antropology and Development", International Social Science Journal. 154. 497-515.

Euben, P. (1989) “Corruption”, Ball, T. Farr, J. y Hansom, R. (ed.) Political Innovation and Conceptiual Change. Cambridge: Cambridge University Press.

Ferguson, J. (1990) The Anti-politics Machine: 'Development', Depoliticisation and Bureaucratic Power in Lesotho. Cambridge: Cambridge University Press.

(1994) 'The Anti-Politics Machine, 'Development' and Bureaucratic Power in Leshoto, The Ecologist, Vol. 24, No. 5, Septiembre/Octubre. 176-181.

Foucault, M. (1978) "Politics and the study of discourse", Ideology and Consciousness, Primavera 1978, No.3.

(1981) "Questions of method: an interview with Michel Foucault", Ideology and Consciousness, Primavera 1981, No.18.

Gadbaw, R.M. y Richards, T. J. (1997) "Anti-corruption as an International Policy Issue: It's Origins, Direction and Implications", Marzo 17, Mimeo.

Gallagher, M. (1991) Rent-Seeking and Economic Growth in Africa. Boulder/Oxford: Westview Press.

Gilpin, R. (1995) "Economic Change and the Challenge of Uncertainty", Ross, R. (ed) East Asia in Transition. Londres: M. e. Sharpe.

Gray, C. y Kaufmann, D. (1998) "Corruption and Development", New perspectives on Combating Corruption. Transparency International and Economic Development Institute of the World Bank.

Gupta, A. (1995) "Blurred Boundaries: The Discourse of Corruption, the Culture of Politics and Imagined State", American Ethnologist 22(2). 375-402.

Klitgaard, R. (1998) “international Cooperation Against Corruption", New Perspectives on Combating Corruption. Transparency International and Economic Development Institute of the World Bank.

Moore, D. B. (1995) "Development Discourse as Hegemony: Toward and Ideological History - 1945-1995”, Moore, D. B. and Schimtz,

Corrupción y desarrollo: Deconsiruyendo el discurso del Banco Mundial 
G. J. (eds.) Debating Development Discourse; Institutional and Popular Perspectives. Londres: Mcmillan Press.

Mosley, H. and Toye (1995) Aid and Power. Londres: Routledge and Kegan Paul.

Munro, P., Lund y Valodia (1999) "The State in a Changing World; Plus Ca Change?: Reflections from the South on the World Bank's 1997 World Development Report", Journal of Internatonal Development, Vol. 11, No.1, Enero-Febrero. 75-91.

Parker, I. (1992) Discourse Dynamics. Londres: Routledge and Kegan Paul.

Rose-Ackerman, S. (1978) Corruption: A Study in Political Economy. New York: Academic Press.

Sachs, W. (ed.) The Development Dictionary. Londres: Zed Books.

Schmitz, G. J. (1995) "Democratization and Demystification: Deconstructing "Governance' as Development Paradigm", Moore, D. and Schmitz, G. Debating Development Discourse; Institutional and Popular Perspectives. Londres: Macmillan.

Tesh, S. (1999) "A Short History of Anti-corruption Activities in the World Bank”, 1818 Society Neweletter, No. 70, Enero-Febrero.

Theobald, R. (1990) Corruption, Development and Underdevelopment. Londres: Macmillan.

Woods, N. (2000) "The Challenge of Good Governance for the IMF and the World Bank Themselves", World Development, Vol. 28, No. 5. 823-841.

World Bank (1989) "Sub-Saharan Africa: From Crisis to Sustainable Growth. A Long-Term Perspective Study”. Washington D. C.: The World Bank.

(1991) "Managing Development: The Governance Dimension. A Discussion Paper". Mimeo.

(1995) "Prohibition of Political Activities in the Bank's Work, Legal Opinion of the General Counsel”, Julio 11.

(1997a) "Should Countries Care About Corruption?" in Annual General Meeting of the World Bank and IMF; Programme of Seminars, Summary of Discussions. Washington D.C.: World Bank.

(1997b) Helping Countries Combat Corruption, a report of the Poverty Reduction and Economic Management Network.

(1997c) World Development Report: The State in a Changing World. Nueva York: Oxford University Press. 